\title{
The way forward
}

\begin{abstract}
Improving maternal health and reducing maternal morbidity and mortality into the future will require a range of health-related and non-health-related strategies. The latter include education for women, better communication, access to transport and technology and cultural change. The role of obstetric medicine practice, research and education in achieving these objectives is discussed in this article. We need to recognise that it will require improvements in health provision and access to achieve our goal of safer childbirth for women and babies throughout the world.
\end{abstract}

@SAGE

\section{Keyword \\ High-risk pregnancy}

Date received: 5 August 2016; accepted: 26 August 2016

Improving maternal health and reducing maternal morbidity and mortality into the future will require a range of health-related and nonhealth-related strategies. The latter include education for women, better communication, access to transport and technology and cultural change. Improvement in these areas has already been recognised as a factor in the successful reduction in maternal mortality in a number of countries such as Bangladesh. ${ }^{1}$ However, as the previous articles in this special issue of Obstetric Medicine have shown, it is improvements in health provision and access which will spearhead our pursuit of safe childbirth for women and babies throughout the world.

All the available data demonstrate the common need for provision of adequate and well-trained staff with appropriate resources to care for women before, during and after pregnancy. If we imagine an ideal world-what part will Obstetric Medicine and the International Society of Obstetric Medicine (ISOM) play in achieving this? It is a basic premise in health provision that evidence-based management which is patient focused and provided by the most appropriate team of healthcare workers will ensure the best outcomes for patients.

For obstetric medicine to be part of this solution for all women, we will first need to establish a more rigorous and expansive research base for our practice. Much of obstetric medicine is currently based on adaptation of non-pregnant research and applying it with knowledge of the specialised biology of pregnancy. Alternatively, there is a reliance on case-based literature, anecdote and experience. There is limited access to properly conducted, randomised trials and a reliance on systematic reviews and meta-analysis. We need to encourage and embrace appropriate trials and share this information, both negative and positive.

The rarity of many of the medical conditions encountered in Obstetric Medicine requires the creation of appropriate global databases and research partnerships. The example of the Global Pregnancy Collaboration should be considered the prototype for future endeavours to promote and enhance research within Obstetric Medicine (https://preempt.cfri.ca/collaboration/global-pregnancy-collaboration). This initiative was launched in 2010 to create international co-operation in pregnancy research by facilitating access to data and biologic samples as well as infrastructure to enhance research particularly in low resource settings.

Similarly, the current ISOM/NASOM listserv provides access for practitioners (with access to the internet) with the opportunity to consult colleagues on rare cases and potentially collect such rare cases into case series' (http://lists.obmed.org/listmanager/listinfo/obmeddiscussion). For this to evolve into a more permanent and useful research tool requires the development of a secure, well-managed database of rare cases in obstetric medicine. The management of such a database should ideally reside with ISOM. This resource would form a living, dynamic record of practitioners experience with various conditions not necessarily described in textbooks or journals.
The development of obstetric medicine to date has been somewhat linear and hierarchical. Individuals with a special interest or opportunity have undertaken practical and/or academic training in this field and have acquired specialist knowledge and experience. This approach has often resulted in territorial disputes and conflict, particularly between obstetricians/maternal-fetal medicine specialists and internist/physicians. Ultimately to impact on the management of maternal medical complications, we need an appropriately well-trained workforce. From the recent Global Obstetric Medicine Survey, it is clear that no one specialty can hold exclusive rights to the practise of obstetric medicine. In each resource setting, the most suitably trained people need to work collaboratively to achieve the best outcome for pregnant women. Such teams in the future are likely to comprise midwives, primary care physicians, obstetric internists/physicians, subspecialist physicians, anaesthetists, paediatricians, intensivists but also medical administrators, public health policy makers and even politicians. The focus of the team must be the pregnant woman and her baby. Until we see all these individuals as part of the team caring for women with medical complications in pregnancy, we will be held back by old-fashioned notions of ownership and parochial interests. Everyone working with women with medical problems in pregnancy needs the opportunity to learn from each other, advance their own training and skills and practice in a patient-focused manner. This approach will be applicable wherever women are having their babies. The form of this training needs to reflect modern approaches to teaching and learning particularly embracing technology and also recognising the importance of interaction with colleagues at conferences. The provision of such training is currently in the hands of the learned Colleges, but the specialty of Obstetric Medicine needs to engage with these educational and training providers to ensure that this subject becomes mandatory as part of physician and obstetric training in the future.

With improved access to information and technology, obstetric medicine has to recognise and relate to the autonomy and empowerment of our patients as we compete with 'Dr Google', patient blogs, interest groups and social media. We need to engage constructively in this field by the provision of suitable consumer material that respects and informs our patients. Equally for practitioners, the creation and provision of such material by ISOM can assist individuals to enhance

Royal Hospital for Women and School of Women's and Children's Health, UNSW, Sydney, Australia

\section{Corresponding author:}

Sandra A Lowe, Royal Hospital for Women and School of Women's and Children's Health, UNSW, Sydney, Australia.

Email: s.lowe@unsw.edu.au 
their patient care. Such information needs to be balanced and acknowledge the desire of women and their families to maintain control over their own health.

As more patients engage with alternative and adjunctive therapies, we need to stay informed and aware of common practices in these areas. In both high- and low-resource settings, our patients are consulting with health practitioners outside our sphere, including traditional healers, naturopaths, traditional Chinese medicine practitioners, homeopaths, chiropractors, etc. In the future, we need to examine which of these practices may be adjunctive and which are harmful to our patients so that we can provide the holistic management they are usually seeking from these practitioners.

Our survey tells us that clinicians are seeking advice on best practice, particularly protocolised guidance for the management of common conditions. This requires appropriate research and collaboration as well as a willingness to compromise to achieve more global approaches to common problems. For example, there are currently numerous National guidelines for the management of hypertensive disease of pregnancy as well as an International guideline published by ISSHP. ${ }^{2}$ Concurrent development of such guidelines by multiple parties is inefficient and potentially unnecessary. This does not negate the recognition that such guidelines need to consider applicability in different resource settings. Again, a globally representative ISOM is well placed to negotiate these challenges.

Every specialty thinks it is the most special but obstetric medicine is the opposite; in many ways, it is the most general, providing the full range of medicine to this specialised population. 'The way forward' is to continually examine where we are and where we should be, always keeping the best interests of our patients foremost.

\section{Declaration of conflicting interests}

The author(s) declared no potential conflicts of interest with respect to the research, authorship, and/or publication of this article.

\section{Funding}

The author(s) received no financial support for the research, authorship, and/or publication of this article.

\section{Ethical approval}

Not applicable.

\section{Guarantor}

SAL.

\section{Contributorship}

Sole author.

\section{References}

1. El Arifeen S, Hill K, Ahsan KZ, et al. Maternal mortality in Bangladesh: a countdown to 2015 country case study. Lancet 2014; 384: 1366-1374.

2. Tranquilli AL, Dekker G, Magee L, et al. The classification, diagnosis and management of the hypertensive disorders of pregnancy: a revised statement from the ISSHP. Pregnancy Hypertens 2014; 4: 97-104. 